\title{
Optical Flux Lattices for Ultracold Atomic Gases
}

\author{
N. R. Cooper \\ Theory of Condensed Matter Group, Cavendish Laboratory, J. J. Thomson Avenue, Cambridge CB3 OHE, United Kingdom
}

(Received 12 January 2011; published 25 April 2011)

\begin{abstract}
We show that simple laser configurations can give rise to "optical flux lattices," in which optically dressed atoms experience a periodic effective magnetic flux with high mean density. These potentials lead to narrow energy bands with nonzero Chern numbers. Optical flux lattices will greatly facilitate the achievement of the quantum Hall regime for ultracold atomic gases.
\end{abstract}

DOI: 10.1103/PhysRevLett.106.175301

PACS numbers: $67.85 . \mathrm{Hj}, 37.10 . \mathrm{Vz}$

One of the most important techniques in the ultracold atom toolbox is the optical lattice [1]: a periodic scalar potential formed from standing waves of light. Optical lattices are central to the use of atomic gases as quantum simulators, and allow the exploration of strong-correlation phenomena related to condensed matter systems $[2,3]$. Their usefulness derives from the fact that the scalar potential has a length scale, set by the optical wavelength $\lambda$, that is similar to the typical interatomic spacing.

Largely separate have been the experimental developments of effective vector potentials, representing the coupling of a charged particle to a magnetic field. A uniform magnetic field can be simulated for neutral atoms using rotation $[1,4,5]$. Gauge fields leading to an effective magnetic field can also be generated by optical dressing [6]. These techniques have allowed experimental studies of quantized vortices in condensed Bose and (paired) Fermi gases $[1,4,7-9]$. An interesting regime of strong correlation, with connections to the fractional quantum Hall effect, is expected when the effective magnetic flux density $n_{\phi}$ is sufficiently high that the length scale $n_{\phi}^{-1 / 2}$ is comparable to the interatomic spacing $[5,10]$. However, the magnetic flux densities achievable using existing techniques are relatively small for large systems $[6,9,11]$, so this strongly correlated regime occurs at very low densities when the gas is weakly interacting and highly susceptible to uncontrolled perturbations $[5,11]$.

In this Letter we describe simple laser configurations that use optical dressing to generate what we refer to as "optical flux lattices." While a conventional optical lattice imprints a periodic scalar potential, an optical flux lattice also imprints a periodic magnetic flux density with nonzero mean, and large magnitude $\bar{n}_{\phi} \sim 1 / \lambda^{2}$. We emphasize that the magnetic flux density is a continuous function of position, so these potentials are distinct from proposed ways to imprint gauge potentials onto deep optical lattices which apply only in the tight-binding limit [12-14]; furthermore, optical flux lattices require only a small number of lasers, so are much easier to implement than these tightbinding proposals. We show that optical flux lattices lead to narrow bands with nonzero Chern numbers [15]. In particular the lowest energy band is topologically equivalent to the lowest Landau level. Since the length scale $\bar{n}_{\phi}^{-1 / 2} \sim \lambda$ is similar to the typical interatomic spacing, optical flux lattices will allow the study of quantum Hall physics at high densities where interaction energy scales are large.

We consider an atom moving in optical fields within the rotating wave approximation [16], with Hamiltonian

$$
\hat{H}=\frac{\hat{\boldsymbol{p}}^{2}}{2 m} \hat{I}+V \hat{M}(\boldsymbol{r})
$$

where $V$ is the energy scale of the optical potential, of dimensionless form $\hat{M}(\boldsymbol{r})$. We focus on two-level systems and write

$$
\hat{M}=\vec{M}(\boldsymbol{r}) \cdot \hat{\vec{\sigma}}=\left(\begin{array}{cc}
M_{z} & M_{x}-i M_{y} \\
M_{x}+i M_{y} & -M_{z}
\end{array}\right)
$$

with $\hat{\vec{\sigma}}$ the vector of Pauli matrices. Additional scalar potentials can be added by conventional optical lattices; for simplicity, we neglect these here. The off-diagonal terms $\left(M_{x, y}\right)$ arise from the optical coupling that effects the interspecies conversion [16]. The diagonal term $\left(M_{z}\right)$ represents a species-dependent potential. One possible implementation of the two-level system is with the ground state and long-lived excited state of an alkaline earth atom or ytterbium [14], in which case $M_{z}$ can be generated by a laser at an "antimagic" wavelength, $\lambda_{\mathrm{am}}$ [14]. As we shall describe below, optical flux lattices can be formed by a standing wave at $\lambda_{\mathrm{am}}$ and three travelling waves of the coupling laser [17]. We shall restrict attention to quasi-two-dimensional (2D) systems with $\boldsymbol{r}=(x, y)$. The resulting flux lattices are readily adapted to $3 \mathrm{D}$, with net flux along one direction.

The emergence of an effective gauge potential is best understood when the kinetic energy is small compared to the energy spacing of the (local) dressed states, obtained from the eigenvalues of $V \hat{M}$. The atom then moves through space adiabatically, staying in a given dressed state. The adiabatic limit is always valid for sufficiently large $V$ provided the spectrum of $\hat{M}$ is nondegenerate. Assuming this to be true (as shall be verified below for the cases of interest), we consider the adiabatic motion in a normalized dressed state 


$$
|\Phi(\boldsymbol{r})\rangle=\left(\begin{array}{l}
\phi_{1}(\boldsymbol{r}) \\
\phi_{2}(\boldsymbol{r})
\end{array}\right) .
$$

Projecting (1) onto adiabatic motion on the state (3) leads to an effective Hamiltonian with both a scalar and a vector potential, the latter given by [6]

$$
q \boldsymbol{A}=i \hbar\langle\Phi \mid \nabla \Phi\rangle
$$

for effective charge $q$. The number density of magnetic flux quanta perpendicular to the $x y$ plane is therefore

$$
n_{\phi} \equiv \frac{q B}{h}=\frac{q}{h} \nabla \times A .
$$

For optical fields of wavelength $\lambda$, it is natural to assume that the vector potential (4) is smoothly varying with $|q \boldsymbol{A}| \lesssim h / \lambda$. Then, the maximum mean flux density in a region of space of sides $L_{x}, L_{y} \gg \lambda$ may be found by applying Stokes' theorem: $\int n_{\phi} d^{2} \boldsymbol{r} \equiv \bar{n}_{\phi} L_{x} L_{y}=(q / h) \times$ $\oint \boldsymbol{A} \cdot d \boldsymbol{r} \lesssim\left(L_{x}+L_{y}\right) / \lambda$, leading to $\bar{n}_{\phi} \lesssim 1 / L \lambda$ with $L=$ $\min \left(L_{x}, L_{y}\right)$. All existing proposals for optically induced gauge fields in the continuum [6], and the scheme implemented in Ref. [9], are of this form with the scale $L$ set by the width of the cloud. Since, typically, $L \gg \lambda$, this leads to relatively small flux density, $\bar{n}_{\phi} \sim 1 / L \lambda$.

Although apparently very general, these considerations neglect the fact that smoothly varying optical fields can induce singularities in $q \boldsymbol{A}$. These singularities depend on the gauge used for (3), and cause no singularities in gaugeinvariant properties. Such issues arise whenever a $U(1)$ gauge field has nonzero flux through a closed manifold, notably leading to Dirac strings for a magnetic monopole. For the optical flux lattices we propose here, there is a net flux through a unit cell which (due to the spatial periodicity) has the topology of a torus. We avoid technical difficulties of the gauge-dependent singularities by defining the local Bloch vector

$$
\vec{n}(\boldsymbol{r})=\langle\Phi(\boldsymbol{r})|\hat{\vec{\sigma}}| \Phi(\boldsymbol{r})\rangle
$$

for which $\vec{n} \cdot \vec{n}=1$. The flux density is then

$$
n_{\phi}=-\frac{1}{8 \pi} \epsilon_{i j k} \epsilon_{\mu \nu} n_{i} \partial_{\mu} n_{j} \partial_{\nu} n_{k} .
$$

This is (minus) the "topological density" of the map from position space, $\boldsymbol{r}=(x, y)$, to the surface of the Bloch sphere, $\vec{n}$ [18]. The number of flux quanta through a region $A$ is $\int_{A} n_{\phi} d^{2} \boldsymbol{r}=\Omega / 4 \pi$ where $\Omega$ is the solid angle that region $A$ maps to on the Bloch sphere. Thus, each time the Bloch vector wraps the surface of the sphere corresponds to one magnetic flux quantum. Optical flux lattices are spatially periodic configurations for which the Bloch vector wraps the sphere a nonzero integer number, $N_{\phi}$, times in each unit cell. The lattice vectors $\boldsymbol{a}_{1}$ and $\boldsymbol{a}_{2}$ are both of order the optical wavelength $\lambda$, so the mean flux density is of order $\bar{n}_{\phi} \sim N_{\phi} / \lambda^{2}$ which is large. We focus on two cases of high symmetry which achieve this goal.
Square lattice.-Consider the optical coupling

$$
\hat{M}_{\mathrm{sq}}=\cos (\kappa x) \hat{\sigma}_{x}+\cos (\kappa y) \hat{\sigma}_{y}+\sin (\kappa x) \sin (\kappa y) \hat{\sigma}_{z}
$$

where $\kappa \equiv 2 \pi / a$. This has square symmetry with $a_{1}=$ $(a, 0), \boldsymbol{a}_{2}=(0, a)$. Achieving this high symmetry in experiment may involve tilting the lasers out of the $x y$ plane to tune the periods of the coupling and the speciesdependent fields [19]. The eigenvalues are nondegenerate at all positions, so the dressed states admit an adiabatic limit. Figure 1(a) shows that, for the lower energy dressed state, the $\left(n_{x}, n_{y}\right)$ components of the Bloch vector exhibit two vortices and two antivortices in the unit cell. These vortices lead to gauge-dependent singularities in the vector potential. However, the Bloch vector varies smoothly, with $n_{z}= \pm 1$ at the vortex cores, in such a way that all four of these regions wrap the sphere in the same sense and contribute a flux density of the same sign. The flux density is shown in Fig. 1(b). It is everywhere non-negative and has total flux $N_{\phi}=2$ in the unit cell. (This may be seen by noting that the two vortices and two antivortices cause $\vec{n}$ to wrap the Bloch sphere twice.) The flux density is not constant, and vanishes at four points in the unit cell. These four points coincide with the locations at which the adiabatic energy is minimum, Fig. 1(b), so form the lattice sites in the tight-binding limit.

Triangular lattice.-An optical flux lattice with triangular symmetry is generated by

$$
\hat{M}_{\text {tri }}=\cos \left(\boldsymbol{r} \cdot \boldsymbol{\kappa}_{1}\right) \hat{\sigma}_{x}+\cos \left(\boldsymbol{r} \cdot \boldsymbol{\kappa}_{2}\right) \hat{\sigma}_{y}+\cos \left(\boldsymbol{r} \cdot \boldsymbol{\kappa}_{3}\right) \hat{\sigma}_{z}
$$

where $\boldsymbol{\kappa}_{1}=(1,0) \kappa, \boldsymbol{\kappa}_{2}=\left(\frac{1}{2}, \frac{\sqrt{3}}{2}\right) \kappa$ and $\boldsymbol{\kappa}_{3}=\boldsymbol{\kappa}_{1}-\boldsymbol{\kappa}_{2}$, with $\kappa \equiv 4 \pi /(\sqrt{3} a)$, giving lattice vectors $\boldsymbol{a}_{1}=$ $(\sqrt{3} / 2,-1 / 2) a$ and $\boldsymbol{a}_{2}=(0,1) a$. Again, the eigenvalues are nondegenerate at all positions. The properties of the

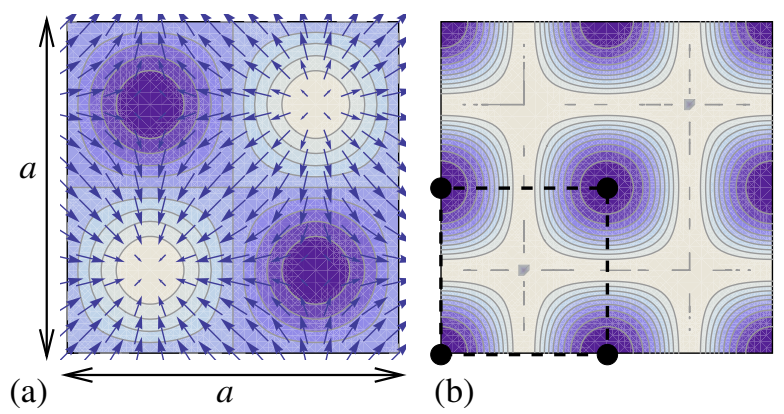

FIG. 1 (color online). Properties of the lower energy dressed state for the square optical flux lattice (8), as a function of position in the unit cell. (a) Local Bloch vector, as represented by the vector $\left(n_{x}, n_{y}\right)$ and contours of $n_{z}$ (light shading for $n_{z}<1$ and dark for $n_{z}>1$ ). (b) The local flux density $n_{\phi}$ is maximal on the lines $x= \pm a / 4$ and $y= \pm a / 4$ and vanishes at four points. (Light (dark) shading indicates large (small) flux density $n_{\phi}$.) The lattice sites and nearest-neighbor hopping in the tightbinding limit are indicated by dark circles and dashed lines. 
lower energy dressed state are shown in Fig. 2. The flux density is maximum on the sites of a honeycomb lattice, and vanishes on the triangular lattice dual to this. The tightbinding limit involves tunneling between the sites of this dual triangular lattice, Fig. 2(b).

The above optical potentials (8) and (9) can be readily generalized to many other cases with nonzero mean flux. (There are also many cases with zero mean, but nonzero local flux density.) The central requirements to generate an optical flux lattice are threefold. First, the coupling laser $\left(M_{x, y}\right)$ must generate optical vortices. A 2D lattice of optical vortices can be formed from a minimum of three travelling waves [20]. The resulting optical field is periodic, with an equal number of (single-winding) vortices $N_{\mathrm{v}}$ and antivortices $N_{\mathrm{av}}$ in a unit cell [21]. Second, the speciesdependent potential $\left(M_{z}\right)$ must be nonzero at the cores of these vortices, such that there is no degeneracy of the dressed states at these points. A small nonzero $M_{z}$ causes the cores of the vortices to have the topology of "merons" [18], in which $\vec{n}(\boldsymbol{r})$ sweeps over half of the Bloch sphere. For a given meron, the sign of $M_{z}$ at its core times the sign of its vorticity determines whether it contributes $+1 / 2$ or $-1 / 2$ a flux quantum. The total number of flux quanta through the unit cell is $N_{\phi}=N_{\mathrm{v}}^{+}-N_{\mathrm{av}}^{+}$, where $N_{\mathrm{v} / \mathrm{av}}^{+}$is the number of vortices or antivortices at which $M_{z}$ is positive. Thus, the third requirement for a nonzero mean flux is that $M_{z}$ varies in space such that $N_{\mathrm{v}}^{+} \neq N_{\mathrm{av}}^{+}$.

We have explored the properties of optical potentials generated by simple laser patterns. An optical flux lattice can be generated using just five travelling waves: three travelling waves of the coupling laser $\left(M_{x, y}\right)$ to effect the vortex lattice, and a standing wave of the speciesdependent potential $\left(M_{z}\right)$. [One such example is to remove one of the four travelling waves from the optical coupling in (9).] In all cases the local flux density is inhomogeneous in space, in some even changing sign. Indeed, one can
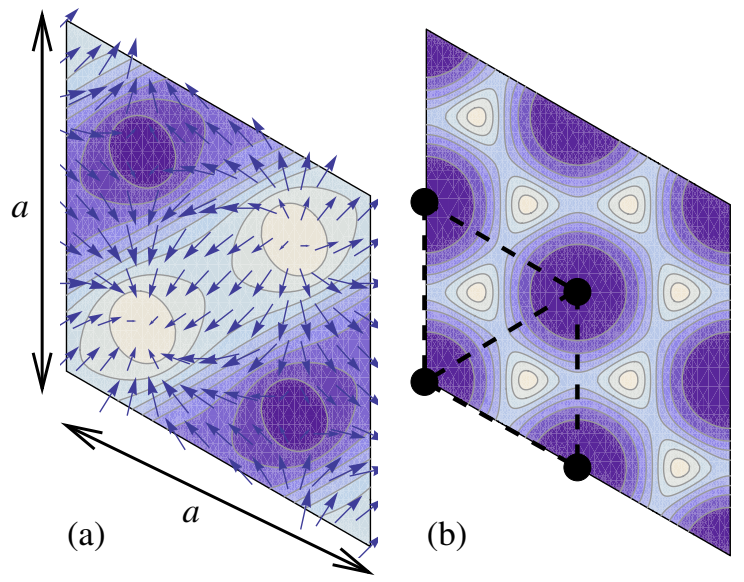

FIG. 2 (color online). (a) Bloch vector and (b) flux density $n_{\phi}$ for the lower energy dressed state of the triangular optical flux lattice (8). The local minima of the adiabatic energy are at the points where $n_{\phi}=0$, forming a triangular lattice in the tightbinding limit (dark circles and dashed lines). show that, for smoothly varying optical fields, the flux density must have at least $N_{\mathrm{v}}+N_{\text {av }}$ zeros in the unit cell [22] The above cases (8) and (9) have non-negative flux density with the minimum number of zeros. For three-, or more-, level systems, an optical flux lattice can have a flux density that nowhere vanishes. We have examples of optical potentials that lead to such cases. However, these require more involved laser configurations, so we do not pursue this here.

Having determined the properties of the optical flux lattices in the adiabatic limit, we now turn to describe their band structures, obtained from the eigenvalues of (1). The laser potentials $\hat{M}_{\mathrm{sq}}$ (8) and $\hat{M}_{\text {tri }}$ (9) are clearly invariant under translations by the respective lattice vectors $\boldsymbol{a}_{1,2}$. In fact, they enjoy higher translational symmetry, being invariant under the unitary transformations

$$
\hat{T}_{1} \equiv \hat{\sigma}_{y} e^{(1 / 2) a_{1} \cdot \boldsymbol{\nabla}} \quad \hat{T}_{2} \equiv \hat{\sigma}_{x} e^{(1 / 2) a_{2} \cdot \boldsymbol{\nabla}}
$$

which effect translations by $\frac{1}{2} \boldsymbol{a}_{1,2}$ and rotations in spin space. These operators do not commute, but satisfy

$$
\hat{T}_{2} \hat{T}_{1}=-\hat{T}_{1} \hat{T}_{2}
$$

This indicates that they represent magnetic translations around a region of space (enclosed by $\frac{1}{2} \boldsymbol{a}_{1}$ and $\frac{1}{2} \boldsymbol{a}_{2}$ ) that contains $1 / 2$ a flux quantum. As is conventional in systems with magnetic translation symmetry [15], we define a magnetic unit cell that encloses an integer number of flux: we choose $\boldsymbol{a}_{1}, \boldsymbol{a}_{2} / 2$. Writing the eigenvalues of the associated (commuting) translation operators $\hat{T}_{1}^{2}$ and $\hat{T}_{2}$ as $e^{i \boldsymbol{k} \cdot \boldsymbol{a}_{1}}$ and $e^{i \boldsymbol{k} \cdot \boldsymbol{a}_{2} / 2}$ defines the Bloch wave vector $\boldsymbol{k}$ and the associated Brillouin zone. The additional symmetry $\hat{T}_{1}$ and the condition (11) cause the energy spectrum $E_{\boldsymbol{k}}$ for all bands to be invariant under $\boldsymbol{k} \cdot \boldsymbol{a}_{2} / 2 \rightarrow \boldsymbol{k} \cdot \boldsymbol{a}_{2} / 2 \pm \pi$ with $\boldsymbol{k} \cdot \boldsymbol{a}_{1} \rightarrow \boldsymbol{k} \cdot \boldsymbol{a}_{1}$.

For the square optical flux lattice (8), a solution of the band structure shows that the lowest energy band does not overlap any higher band for $V \gtrsim 0.1 \hbar^{2} \kappa^{2} / m$. The Chern number [15] of this band is 1 , the sign being reversed under an odd number of sign changes to the terms in (8). Thus, the lowest energy band is topologically equivalent to the lowest Landau level of a charged particle in a uniform magnetic field. It is instructive to consider the band structure for $V \gg \hbar^{2} \kappa^{2} / m$, when the variation in the adiabatic energy is dominant, and the low energy bands are well described by a tight-binding model [2]. The minima of the adiabatic potential form a square lattice, Fig. 1(b). Nearestneighbor hopping on this square lattice leads to a model in which each plaquette encloses $1 / 2$ a flux quantum. (Note that the tight-binding lattice has four plaquettes per $a \times a$ unit cell, for which $N_{\phi}=2$.) The magnetic unit cell contains two lattice sites, so there are two tight-binding bands. The bands touch at two Dirac points [23], so one can speak only of the Chern number of the two bands together. This total Chern number is zero, consistent with the fact that this nearest-neighbor tight-binding model is time-reversal 


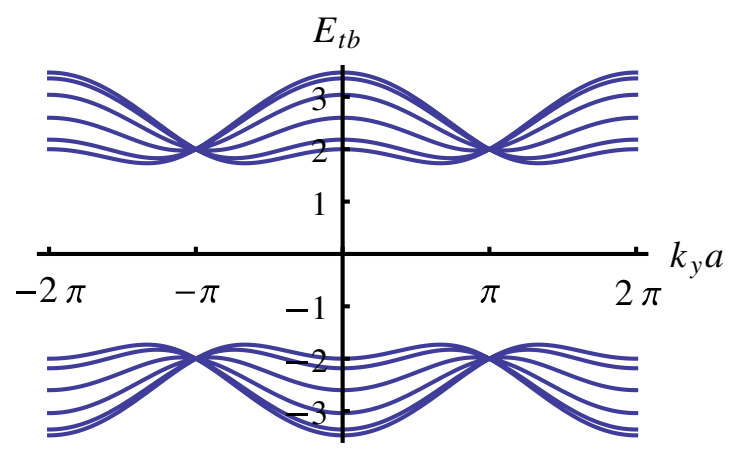

FIG. 3 (color online). Lowest energy bands for the triangular flux lattice (9) in the nearest-neighbor tight-binding limit (for uniform spacing of $-2 \pi / \sqrt{3} \leq k_{x} a \leq 2 \pi / \sqrt{3}$ ). The energy $E_{\mathrm{tb}}$ is relative to the atomic limit, in units of the nearest-neighbor hopping. The bands have Chern numbers \pm 1 .

symmetric [24]. In the physical model, with $m V / \hbar^{2} \kappa^{2}$ large but finite, time-reversal symmetry is broken by next nearest-neighbor hopping across diagonals of the square lattice. This leads to closed loops around plaquettes which contain $1 / 4$ of a flux quantum. This perturbation acts to split the bands at the two Dirac points, and the two bands acquire Chern numbers of \pm 1 .

The band structure of the triangular optical flux lattice (9) has the same qualitative properties, the lowest energy band having a Chern number of 1 . In this case, timereversal symmetry is broken even in the tight-binding limit with nearest-neighbor hopping. The energy minima are at the sites of a triangular lattice, Fig. 2(b), the elementary plaquettes of which enclose $1 / 4$ of a flux quantum. The energy spectrum of the resulting tight-binding model, shown for a convenient gauge in Fig. 3, has two narrow bands that are well separated in energy and have Chern numbers of \pm 1 .

Optical flux lattices will allow experiments on ultracold gases to explore many very interesting phenomena. Since they lead to a lowest energy band with a nonzero Chern number, noninteracting fermions filling this band (with one fermion per magnetic unit cell) will exhibit the integer quantum Hall effect. Signatures of the resulting chiral edge state could be observed in the collective modes, which will rotate with a handedness determined by the sign of the Chern number. The square lattice in the nearest-neighbor tight-binding limit also offers the possibility to study fermionic Dirac physics. Within mean-field theory, interacting bosons loaded into the chiral band will develop vortex lattices with very high flux density [25]. Owing to the very high flux density, it should be possible to reach a regime where the $2 \mathrm{D}$ boson density is comparable to the mean flux density, $\bar{n}_{\phi} \sim 1 / \lambda^{2}$, where strongly correlated fractional quantum Hall states of bosons $[5,10]$ or related states on lattices [26] can appear. A leading candidate is the $\nu=1 / 2$ bosonic Laughlin state on the triangular lattice (9), for which the lowest energy chiral band is narrow and well separated from higher bands. There is $1 / 2$ a flux quantum per lattice site, so the Laughlin state appears at $1 / 4$ filling. It will be interesting also to explore strong correlation phenomena in 3D settings, with an optical flux lattice providing net flux in one direction. Thus, optical flux lattices will allow cold atom systems to be used to study interesting phenomena related to the quantum Hall effects. In contrast to electronic systems, these systems will allow tuning of interparticle interactions and explorations of bosonic variants and lattice effects.

I am grateful to Jean Dalibard for many helpful comments. This work was supported by EPSRC Grant No. EP/ F032773/1

[1] I. Bloch, J. Dalibard, and W. Zwerger, Rev. Mod. Phys. 80, 885 (2008).

[2] D. Jaksch et al., Phys. Rev. Lett. 81, 3108 (1998).

[3] M. Greiner et al., Nature (London) 415, 39 (2002).

[4] A. L. Fetter, Rev. Mod. Phys. 81, 647 (2009).

[5] N. R. Cooper, Adv. Phys. 57, 539 (2008).

[6] J. Dalibard, F. Gerbier, G. Juzeliūnas, and P. Öhberg, arXiv:1008.5378.

[7] K. W. Madison, F. Chevy, W. Wohlleben, and J. Dalibard, Phys. Rev. Lett. 84, 806 (2000).

[8] M. W. Zwierlein et al., Nature (London) 435, 1047 (2005).

[9] Y.-J. Lin et al., Nature (London) 462, 628 (2009).

[10] N. R. Cooper, N. K. Wilkin, and J. M. F. Gunn, Phys. Rev. Lett. 87, 120405 (2001).

[11] V. Schweikhard et al., Phys. Rev. Lett. 92, 040404 (2004).

[12] D. Jaksch and P. Zoller, New J. Phys. 5, 56 (2003).

[13] E. J. Mueller, Phys. Rev. A 70, 041603 (2004).

[14] F. Gerbier and J. Dalibard, New J. Phys. 12, 033007 (2010).

[15] D. J. Thouless, M. Kohmoto, M. P. Nightingale, and M. den Nijs, Phys. Rev. Lett. 49, 405 (1982).

[16] C. Cohen-Tannoudji, J. Dupont-Roc, and G. Grynberg, Atom-Photon Interactions (Wiley, New York, 1992).

[17] For other implementations involving coupling via twophoton transitions (e.g., for hyperfine levels), one needs lasers that allow three different momentum transfers.

[18] S. M. Girvin, in Topological Aspects of Low Dimensional Systems, edited by A. Comtet, T. Jolicoeur, S. Ouvry, and F. David (Springer-Verlag, Berlin, 2000).

[19] For $\mathrm{Yb}$ these natural periods are $\lambda_{0}=578 \mathrm{~nm}$ and $\lambda_{\mathrm{am}} / 2=560 \mathrm{~nm}$, respectively [14].

[20] J. Masajada and B. Dubik, Opt. Commun. 198, 21 (2001).

[21] Defining the three wave vectors $\boldsymbol{k}_{1,2,3}$, the reciprocal lattice vectors of this cell are $\boldsymbol{k}_{2}-\boldsymbol{k}_{1}$ and $\boldsymbol{k}_{3}-\boldsymbol{k}_{1}$.

[22] The proof follows by noting that $n_{\phi}=0$ at the saddle points of $n_{z}(\boldsymbol{r})$. From Morse theory applied to $n_{z}$ on the unit cell, the number of saddle points is the sum of the numbers of maxima and minima, a sum at least as large as $N_{\mathrm{v}}+N_{\mathrm{av}}$.

[23] L.-K. Lim, C. M. Smith, and A. Hemmerich, Phys. Rev. Lett. 100, 130402 (2008).

[24] Each plaquette contains $1 / 2$ a flux quantum, which is gauge equivalent to $-1 / 2$ a flux quantum.

[25] G. Möller and N. R. Cooper, Phys. Rev. A 82, 063625 (2010).

[26] G. Möller and N. R. Cooper, Phys. Rev. Lett. 103, 105303 (2009). 